\title{
Treatment Challenges of Primary Acinic Cell Carcinoma of the Breast
}

\author{
Sundus Abbasia, b, c, Lisa Hopkins ${ }^{\mathrm{a}, \mathrm{b}}$
}

\begin{abstract}
We present a rare case of primary acinic carcinoma in the breast, which is almost always associated with salivary glands. We will discuss the multidisplinary approach to treatment, highlighting the role of chemotherapy and radiation. Additionally, this tumor was seen on magnetic resonance imaging (MRI), which is not a readily used diagnostic test for breast disease, emphasizing its diagnostic sensitivity in these patients.
\end{abstract}

Keywords: Acinic carcinoma; Breast cancer; Treatment

\section{Introduction}

Acinic cell carcinoma (ACC) is a low grade malignancy unique to salivary gland treated with a combination of surgical and oncologic management. Although the treatment plan for salivary malignancy is well established, primary breast acinic carcinoma remains a challenge to the multidisplinary team with various treatment algorithms. We hope this paper will raise awareness of this rare subtype and direct a treatment plan for similar patients in the future.

\section{Case Report}

We present a 57-year-old female with an abnormal routine mammogram. Her history is significant for maternal breast cancer. Her ultrasound showed no abnormality and mammogram showed a $2 \mathrm{~cm}$ focal asymmetry, classified as BI-RADS 0 (not diagnostic). Subsequently a magnetic resonance imaging (MRI) was preformed that showed a right breast lobulated enhancing mass which when biopsied showed ACC. The

Manuscript submitted December 26, 2018, accepted January 15, 2019

aDepartment of Surgery, Saint Joseph's Regional Medical Center, Paterson, NJ, USA

bDepartment of Breast Oncology, Saint Peter's Medical Center, New Brunswick, NJ, USA

${ }^{\mathrm{c} C}$ Corresponding Author: Sundus Abbasi, 56 Franklin P1, Apt 3, Summit, NJ 07901, USA. Email: Sundus.abbasi@gmail.com,

doi: https://doi.org/10.14740/jcs373 patient was taken for breast conserving therapy with axillary node dissection. Pathology confirmed ACC, estrogen negative, progesterone positive, HER-2 negative with negative margins and no positive lymph nodes. Of note, she was progesterone positive in a typically triple negative cancer. After discussion with the radiation oncology team, she was deemed not a candidate for adjuvant chemotherapy but did receive 21 cycles of adjuvant radiotherapy. On 5-year follow-up, she has had no locoregional reoccurrence or metastatic disease.

\section{Discussion}

About one in eight (12.4\%) of women will develop invasive breast cancer over the course of their lifetime with greater than 250,000 new cases diagnosed annually [1]. Common malignant subtypes include invasive ductal carcinoma and invasive lobular carcinoma with varying hormone susceptibility. Rare subtypes pose a challenge to common treatment algorithms. The breast functionally resembles a secretory gland and although this subtype is rare, it is explanatory. ACC is usually a triple-negative breast carcinoma that demonstrates morphologic similarity with primary acinic cell carcinoma of the breast, but with less studied treatment [2]. Our patient was progesterone positive on hormonal analysis, unlike the typical presentation. Although this did not impact her morbidity or mortality, as ACC is considered a low-grade tumor.

The first case of ACC of the breast was described by Roncaroli in 1996, since then only 39 cases have been documented [3]. The mean age of patients ranged from 20 - 80 years of age, with 51 as the most common. Although the histopathological features remain similar for the 39 documented cases, the treatment remains unclear. In a retrospective analysis 29 patients were treated with modified radical mastectomy, 16 were treated with breast conservation therapy, eight of which had a sentinel lymph node dissection, four of the modified radical and sentinel node patients received neoadjuvant chemotherapy, 10 breast conservatory and modified radial patients received adjuvant chemotherapy, and six of the breast conservatory patients received radiation therapy [4]. Locoregional reoccurrence only happened in one patient after lumpectomy with no neoadjuvant or adjuvant chemotherapy or radiation therapy, and three patients had metastasis after surgery with neoadjuvant chemotherapy and radiation therapy [4]. Unfortunately, the articles discuss their decision-making regarding which patients receive neoadjuvant or adjuvant chemotherapy or radiation therapy. The treatment plans seems to be based on a case-by-case ba- 
sis, emphasizing the need for a more structured treatment algorithm.

Another treatment challenge is the radiographic variability. Typically ACC will present with lucency or scattered calcifications on mammogram, much like invasive ductal carcinoma [4]. In some patients, like this case report, the mammogram will show only a focal asymmetry, which was considered BI-RADS 0 and not diagnostic. MRI was more sensitive in detecting this suspicious lesion and may be more useful in similar patients.

\section{Conclusions}

Salivary gland tumors are rare triple negative cancers of the breast and the degree of variability in treatment plans continues to become a challenge for the multidisplinary breast oncology team. We hope this paper raises awareness of this rare subtype and helps direct a similar treatment plan among breast surgeons and ultimately a standard treatment algorithm.

\section{References}

1. Breastcancer.org. U.S. Breast Cancer Statistics. https:// www.breastcancer.org/symptoms/understand_bc/statistics.

2. Conlon N, Sadri N, Corben AD, Tan LK. Acinic cell carcinoma of breast: morphologic and immunohistochemical review of a rare breast cancer subtype. Hum Pathol. 2016;51:16-24.

3. Roncaroli F, Lamovec J, Zidar A, Eusebi V. Acinic cell-like carcinoma of the breast. Virchows Arch. 1996;429(1):6974.

4. Limite G, Di Micco R, Esposito E, Sollazzo V, Cervotti M, Pettinato G, Varone V, et al. Acinic cell carcinoma of the breast: review of the literature. Int J Surg. 2014;12(Suppl 1):S35-39. 